\title{
Ultrafast Synthesis of Ni-MOF in One Minute by Ball Milling
}

\author{
Ren Zhang ${ }^{1,2,+}$, Cheng-An Tao ${ }^{2, *}+{ }^{+}$, Rui Chen ${ }^{2}$, Lifang Wu ${ }^{1,2}$, Xiaoxuan Zou ${ }^{1, *}$ \\ and Jianfang Wang $2, *$ (D) \\ 1 College of Chemistry, Key Laboratory of Environmental Friendly Chemistry and Application in Ministry of \\ Education, Xiangtan University, Xiangtan 411105, China; 13574092586@163.com (R.Z.); \\ wulifangyou@163.com (L.W.) \\ 2 College of Liberal Arts and Science, National University of Defense Technology, Changsha 410073, China; \\ Chenrui13@nudt.edu.cn \\ * Correspondence: tca02@mails.thu.edu.cn (C.-A.T.); zouxiaoxuanxtu@163.com (X.Z.); \\ wangjianfang@nudt.edu.cn (J.W.); Tel.: +86-731-8700-1822 (C.-A.T.); +86-731-8700-1801 (J.W.) \\ + These authors contributed equally to this work.
}

Received: 28 November 2018; Accepted: 16 December 2018; Published: 18 December 2018

\begin{abstract}
A mechanical ball milling method for ultrafast synthesis of a nickel-based metal organic framework (Ni-MOF) has been proposed. The Ni-MOF was successfully synthesized in merely one minute without any solvent, additives, or preliminary preparation. The effect of milling time, mechano-frequency, type of assistant liquid, and amount of assistant water were systematically explored. It was found that the product can be obtained even only at a mechano-frequency of $10 \mathrm{~Hz}$ within one minute without any external solvent-assist, which indicated that the crystal water present in the nickel precursor was sufficient to promote MOF formation. Increasing the milling time, raising the mechano-frequency, and the addition of assistant solvent could promote the reaction and increase the yield. The method is rapid, highly efficient, eco-friendly, and has great scalability. The product generated within merely one minute even exhibited high capacitance.
\end{abstract}

Keywords: metal-organic frameworks; ball milling; solvent-free; mechanochemistry; liquid assisted grinding

\section{Introduction}

Metal-organic frameworks (MOFs) [1,2] have attracted intense attention during the past two decades due to their intriguing structural properties, which led to many potential applications, including gas storage [3], separations [4], catalysis [5], and sensing [6-8], etc. Recently, MOFs have been proven to be useful for electrochemical energy storage and considered as potential electrode material for supercapacitors because of their very large surface area, adjustable pore size, controllable microporous structure, and special structures with potential pseudo-capacitive redox centers [9-12].

Among them, nickel-based MOFs have get more interest not only because they have been demonstrated to have great application prospects for supercapacitors, but also because they could be the template/precursor for unique metal oxide and carbon materials with specific structure, especially the $\mathrm{Ni}_{3}(\mathrm{BTC})_{2} \cdot 12 \mathrm{H}_{2} \mathrm{O}$ (BTC $=1,3,5$-benzenrtricarboxylic acid). Kong's group [13] demonstrated that the hydrothermally-synthesized $\mathrm{Ni}_{3}(\mathrm{BTC})_{2} \cdot 12 \mathrm{H}_{2} \mathrm{O}$ had high specific capacitance of $726 \mathrm{~F} / \mathrm{g}$. Wang et al. [14] took the Ni-MOF as a precursor to prepare mesoporous metal oxide by calcining the precursor in the air, and the prepared nickel oxide $(\mathrm{NiO})$ had high-capacitance retention at high scan rate and exhibited excellent cycle-life stability due to its special mesoporous character with bimodal size distribution. Chen et al. [15] synthesized large-scale of multiwalled carbon nanotubes 
using $\mathrm{Ni}_{3}(\mathrm{BTC})_{2} \cdot 12 \mathrm{H}_{2} \mathrm{O}$ as precursor. However, the synthesis processes of Ni-MOFs normally require bulk solvents, high temperatures, and long reaction times. For instance, Wang et al. [14] synthesized $\mathrm{Ni}_{3}(\mathrm{BTC})_{2} \cdot 12 \mathrm{H}_{2} \mathrm{O}$ in a Teflon-lined autoclave and heated at $200{ }^{\circ} \mathrm{C}$ for $24 \mathrm{~h}$, Du et al. [16] synthesized Ni-BTC MOF in a Teflon-lined autoclave and heated to $150{ }^{\circ} \mathrm{C}$ for $12 \mathrm{~h}$ in $\mathrm{N}, \mathrm{N}$-dimethyl formamide (DMF), and Kong's group [13] prepared Ni-MOF at a lower temperature of $105^{\circ} \mathrm{C}$ in DMF but for longer time (up to two days). Although Jin et al. [17] described a simple solution-phase method for the synthesis of $\mathrm{Ni}_{3}(\mathrm{BTC})_{2} \cdot 12 \mathrm{H}_{2} \mathrm{O}$ under room temperature in a short time, an organic linker must be deprotonated, and an organic solvent was also used. Therefore, a simple, green, rapid, and energy-efficient route to generate Ni-based MOFs without high temperatures or bulk solvents is still highly desirable.

Mechanochemistry, i.e., chemical synthesis enabled or sustained by mechanical force [18], has been recently introduced as an alternative to conventional MOF syntheses $[19,20]$. Mechanochemical synthesis of MOFs enable avoiding bulk solvents, high temperature, and/or corrosive reagents frequently employed in solution synthesis. Importantly, it is even possible to be more rapid and efficient than solvent-based methods. Since the synthesis of the moderately porous copper(II) isonicotinate MOF in pioneering work reported by James' group [21], milling procedures have been successfully applied for the synthesis of several popular MOF materials, such as zeolitic azolate frameworks [22,23], rare-earth(III) metal-organic frameworks [24], isoreticular metal-organic frameworks (IRMOFs) [25], iron(III) trimesate MIL-100 (MIL = Materials of Institut Lavoisier) [26], MOF-74 [27], Hong Kong University of Science and Technology (HKUST)-1 [28], $\mathrm{Cu}_{2} \mathrm{I}_{2}$ (triphenylphosphine) ${ }_{2}(\mathrm{~L})_{\mathrm{n}}(n=1,2)$ [29], copper-based MOF-505 [30], and UiO-66 (UiO = University of Oslo) and UiO-67 derivatives [31-33]. Previously, Pichon and James [21] described a survey of the potential reactions between $\mathrm{Ni}(\mathrm{OAc})_{2}$, $\mathrm{Ni}\left(\mathrm{NO}_{3}\right)_{2}, \mathrm{NiSO}_{4}$, and $\mathrm{H}_{3} \mathrm{BTC}$ under mechanochemical solvent-free conditions and, unfortunately, only grinding nickel sulfate with $\mathrm{H}_{3} \mathrm{BTC}$ gave a partial reaction. Consequently, the ability of mechanochemistry to access Ni-MOFs rapidly, has remained insufficient explored.

Herein, we report the ultrafast synthesis of Ni-MOF by a mechanical ball milling method (Figure 1). Notably, Ni-MOF could be obtained in merely one minute without bulk solvent, additives, or any preliminary preparation. The structure and the morphology of Ni-MOF were confirmed by powder X-ray diffraction (PXRD), and scanning electron microscopy (SEM), respectively. Interestingly, the Ni-MOF was formed by fast crystallization within one minute, after which there was no apparent change in the yield and crystallinity, even after $180 \mathrm{~min}$. The effect of milling time, mechano-frequency, kind of assistant liquid, amount of assistant water were systematically explored. The scalability of the ball milling method were also investigated. The generated Ni-MOFs exhibit high capacitance as high as $640 \mathrm{~F} / \mathrm{g}$ at the current density of $1 \mathrm{~A} / \mathrm{g}$. To the best of our knowledge, this is the first example of ultrafast synthesis of Ni-MOF by ball milling.

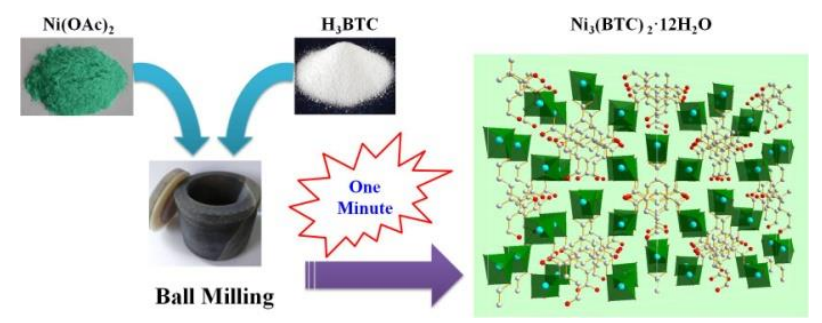

Figure 1. Schematic representation of the ball milling method for the rapid synthesis of Ni.MOF.

\section{Materials and Methods}

\subsection{Materials}

Nickel(II) acetate tetrahydrate $\left[\mathrm{Ni}(\mathrm{OAc})_{2} \cdot 4 \mathrm{H}_{2} \mathrm{O}, \mathrm{AR}\right]$ was purchased from Tianjin Fenchuan Technology Co. Ltd. (Tianjin, China) and benzene-1,3,5-tricarboxylic acid $\left(\mathrm{H}_{3} \mathrm{BTC}, 99 \%\right)$ was purchased from J\&K Scientific Ltd. (Beijing, China). DMF(AR) was purchased from Tianjin Hengxing Chemical 
Preparation Co. Ltd. (Tianjin, China), methanol (MeOH, AR) was purchased from Sinopharm Chemical Reagent Co. Ltd. (Beijing, China), and ethanol (EtOH, 99.7\%) was purchased from General-Reagent (Shanghai, China). Pure water was homemade.

\subsection{Synthesis of $N i-M O F$}

Ni-MOFs were synthesized by ball milling of of 2:3 (molar ratio) mixtures of $\mathrm{H}_{3} \mathrm{BTC}$ and $\mathrm{Ni}(\mathrm{OAc})_{2} \cdot 4 \mathrm{H}_{2} \mathrm{O}$ at varied frequency from $10 \mathrm{~Hz}$ to $50 \mathrm{~Hz}$. In a typical reaction, about one gram of the H3BTC and metal precursor mixture was ball milled for various times $(1,5,30,60$, and $180 \mathrm{~min})$ in a agate vial $(80 \mathrm{~mL})$ using a QM-QX0.4L mill (Miqi Instrument Equipment Co., Ltd., Changsha, China) with the addition of specific amount of water $(0,0.5,1$, or $2 \mathrm{~mL})$ or $1 \mathrm{~mL}$ of other organic solvent $(\mathrm{MeOH}, \mathrm{EtOH}$, and DMF) and increased the feed rate by three times and five times. The ball-to-powder mass ratio was consistently kept at about 5:1 for all experiments. The products were scraped off the jar walls, washed with water and ethanol trice, then the supernatant was removed by centrifugation, and the solid product was dried at $60^{\circ} \mathrm{C}$ in an oven for $12 \mathrm{~h}$. The enlarged scale reaction was performed with three or five times of the $\mathrm{H}_{3} \mathrm{BTC}$ and metal precursor mixture. The yield was calculated according to the following equation based on the number of moles of $\mathrm{Ni}(\mathrm{II})$.

$$
\text { Yield }=\frac{n_{\mathrm{Ni}(\mathrm{II}) \text { in } \mathrm{Ni}-\mathrm{btc}}}{n_{\mathrm{Ni}(\mathrm{OAc})_{2}}} \times 100 \%
$$

\subsection{Characterization}

FTIR spectra were recorded in the range of $400-4000 \mathrm{~cm}^{-1}$ on a PerkinElmer Spectra Two FT-IR spectrophotometer (Waltham, MA, USA) with an attenuated total reflectance (ATR) accessory. The milled samples were analyzed by powder XRD on a Tri III powder diffractometer (Rigaku, Tokyo, Japan) using $\mathrm{Cu} \mathrm{K} \alpha$ radiation between $8^{\circ}$ and $60^{\circ}$ with a scan rate of $5^{\circ} / \mathrm{min}$. Thermogravimetric analyses (TGA) were performed on a STA6000 thermal analyzer (PerkinElmer, Waltham, MA, USA) under $\mathrm{N}_{2}$ with a heating rate of $4{ }^{\circ} \mathrm{C} / \mathrm{min}$. Nitrogen adsorption-desorption isotherms, pore size distributions and surface areas of the samples were measured via $\mathrm{N}_{2}$ adsorption-desorption at 77 K on a BEL SORP-mini II surface area and porosity analyzer (Bel Japan Inc., Osaka, Japan). Before measurement, the samples were activated at $60{ }^{\circ} \mathrm{C}$ for $12 \mathrm{~h}$. The morphology of the sample was observed by a SEM Model S-4800 (Hitachi, Tokyo, Japan).

All electrochemical measurements were done in a three-electrode experimental setup. A platinum wire electrode and a saturated $\mathrm{Ag} / \mathrm{AgCl}$ electrode were used as the counter and reference electrodes, respectively. All the electrochemical measurements were carried out in $6 \mathrm{~mol} / \mathrm{L} \mathrm{KOH}$ aqueous electrolyte using a CHI660C (Shanghai Chenhua Apparatus, Shanghai, China) electrochemical workstation.

\section{Results and Discussion}

\subsection{Synthesis of $\mathrm{Ni}-\mathrm{MOF}$}

\subsubsection{Effect of Grinding Time}

Firstly, the $\mathrm{Ni}-\mathrm{MOF} \mathrm{Ni}_{3}(\mathrm{BTC})_{2} \cdot 12 \mathrm{H}_{2} \mathrm{O}$ was synthesized by grinding of $2: 3$ (molar ratio) mixtures of $\mathrm{H}_{3} \mathrm{BTC}$ and $\mathrm{Nickel}(\mathrm{II})$ acetate tetrahydrate $\left[\mathrm{Ni}(\mathrm{OAc})_{2} \cdot 4 \mathrm{H}_{2} \mathrm{O}\right.$ ] with water-assist under mechano-grinding at $50 \mathrm{~Hz}$ for a specific time $(1,5,30,60$, and $180 \mathrm{~min})$. The products are denoted as Ni-BTC-1 m, Ni-BTC-5 m, Ni-BTC-30 m, Ni-BTC-60 m, and Ni-BTC-180 m, respectively. There is no apparent change in the yield, which only varied from $66 \%$ to $72 \%$ (Table S1). The Fourier transform infrared (FTIR) spectra of different Ni-MOFs are very similar. These spectra clearly show the vibrational bands of the waters around 3500 and $3200 \mathrm{~cm}^{-1}$, which suggests there are crystallization waters in the product (Figure 2a). Additionally, there is no band at around $1710 \mathrm{~cm}^{-1}$ (which is the characteristic of protonated carboxylic groups) observed in Ni-MOFs curves, suggesting the absence of protonated carboxylic groups in the product. 
The XRD pattern shows the characteristic diffraction peaks of $\mathrm{Ni}_{3}(\mathrm{BTC})_{2} \cdot 12 \mathrm{H}_{2} \mathrm{O}$ (Figure $2 \mathrm{~b}$ ), which matches well with the result of the reported literature $[14,16,17,34]$, and the simulated pattern for $\left[\mathrm{Ni}_{3}(\mathrm{BTC})_{2} \cdot 12 \mathrm{H}_{2} \mathrm{O}\right]$ was based on single-crystal data from the Cambridge Crystallographic Data Centre (CCDC) [35]. The generated product in only one minute has already shown good crystallinity. To evaluate the effect of grinding time on the size of Ni-MOF crystals, the full width at half maxima of peaks (FWHM) at $17.7^{\circ}$ corresponding to the (220) lattice plane were listed in Table S2. The average size of Ni-MOF crystals was inversely proportional to the FWHM. The FWHM decreases slightly, at first, and then increases, and finally decreases along with grinding time, indicating that the average size of Ni-MOF crystals changed slightly along with the grinding time.

(a)

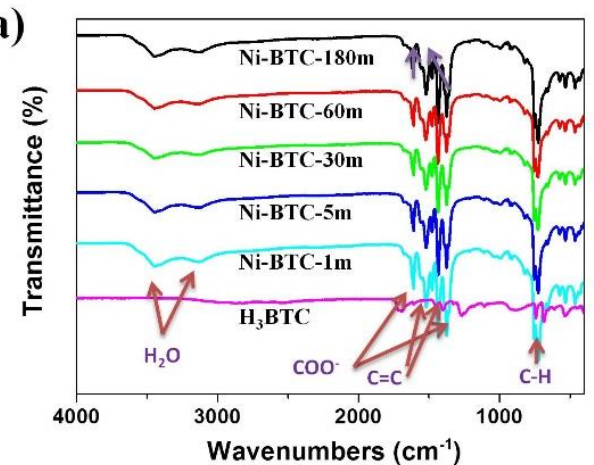

(c)

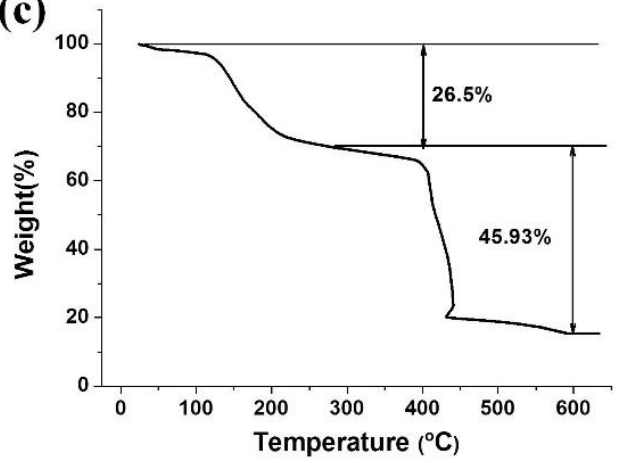

(b)

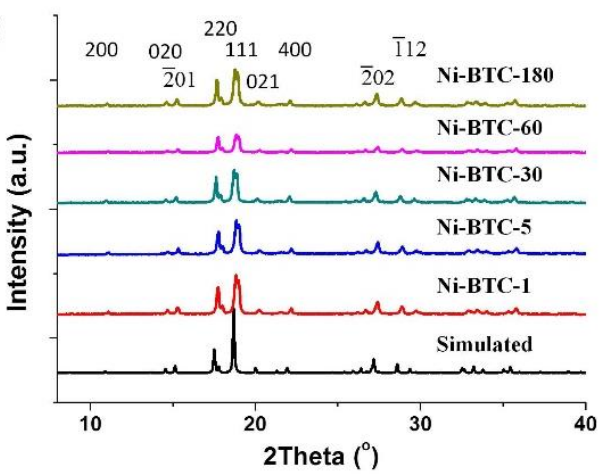

(d)

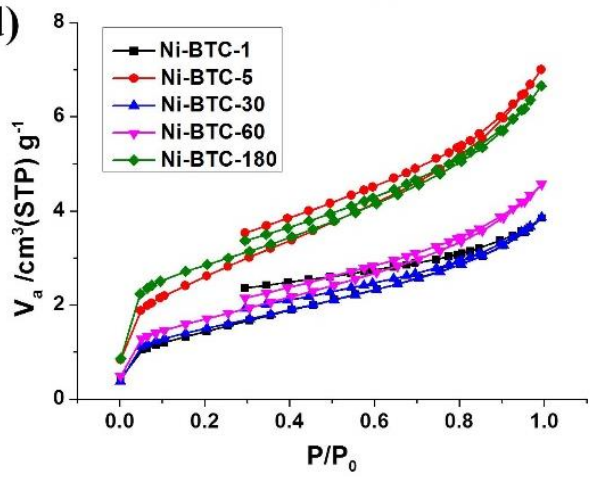

Figure 2. Characterizations of the Ni-BTC samples obtained at various reaction times. (a) FTIR spectra, (b) XRD patterns, (c) TGA curve of Ni-BTC-1m, and (d) Nitrogen adsorption and desorption.

To further confirm the composition of the Ni-MOF, the thermogravimetric analyses (TGA) were performed under air with a heating rate of $4{ }^{\circ} \mathrm{C} / \mathrm{min}$. There are two different stages of weight loss in the TGA curve of Ni-MOF-1m, as shown in Figure 2c. In the first stage, weight loss of $27.35 \mathrm{wt} \%$ from $100{ }^{\circ} \mathrm{C}$ to $250{ }^{\circ} \mathrm{C}$ could be ascribed to the loss of crystallization water molecules. The second sharp weight loss started from $250{ }^{\circ} \mathrm{C}$ and ended at $400{ }^{\circ} \mathrm{C}$, due to the decomposition of the organic frameworks, and the final residue was $\mathrm{NiO}$ [17]. These evidences further prove that the coordinated or adsorbed $\mathrm{H}_{2} \mathrm{O}$ molecules existence in the Ni-MOFs. This result is in conformity to the FTIR data. Additionally, the weight loss is in agreement with the chemical formula $\mathrm{Ni}_{3}\left(\mathrm{C}_{6} \mathrm{H}_{3}(\mathrm{COO})_{3}\right)_{2} \cdot 12 \mathrm{H}_{2} \mathrm{O}$.

In addition, nitrogen adsorption-desorption isotherms, the specific surface area and pore structures of Ni-MOFs were studied by surface area and porosity analyzer, and the results are shown in Figure $2 \mathrm{~d}$ and Table S3. The Brunauer-Emmett-Teller (BET) specific surface area of Ni-MOF- $1 \mathrm{~m}$ is only $4.85 \mathrm{~m}^{2} / \mathrm{g}$, after the longer reaction time, the specific surface area of Ni-MOF-180m reaches the maximum of $10.08 \mathrm{~m}^{2} / \mathrm{g}$. The average pore sizes of Ni-MOFs are about $4.6 \mathrm{~nm}$ except Ni-MOF-180m $(4.1 \mathrm{~nm})$. According to the Barrett-Joyner-Halenda (BJH) analysis of the Ni-BTC samples obtained at various reaction times, as shown in Figure S1, the pore sizes in the micropore range are around $1.7 \mathrm{~nm}$ for all MOF samples. However, the pore sizes in the mesopore range are different, and it is inferred 
that the porosity comes from the interparticle voids, which is different due to the varied morphology of the MOFs.

Morphological investigations of Ni-MOFs were carried out using a field-emission SEM, and the SEM images are shown in Figure 3. Ni-MOF-1 m exhibits rod-like shapes, but the dispersion of their sizes is very broad. The larger ones have a length and width of $10 \mu \mathrm{m}$ and $500 \mathrm{~nm}$, respectively, while the smaller ones have a length and width of about $500 \mathrm{~nm}$ and $100 \mathrm{~nm}$ (Figure 3a,b). The surface of microcrystals of Ni-MOF-1 m looks very smooth. For the products with longer reaction time, there are no apparent change in the morphology (Figures $3 c-f$ and S2), suggesting the success of synthesis of Ni-MOF in merely one minute.
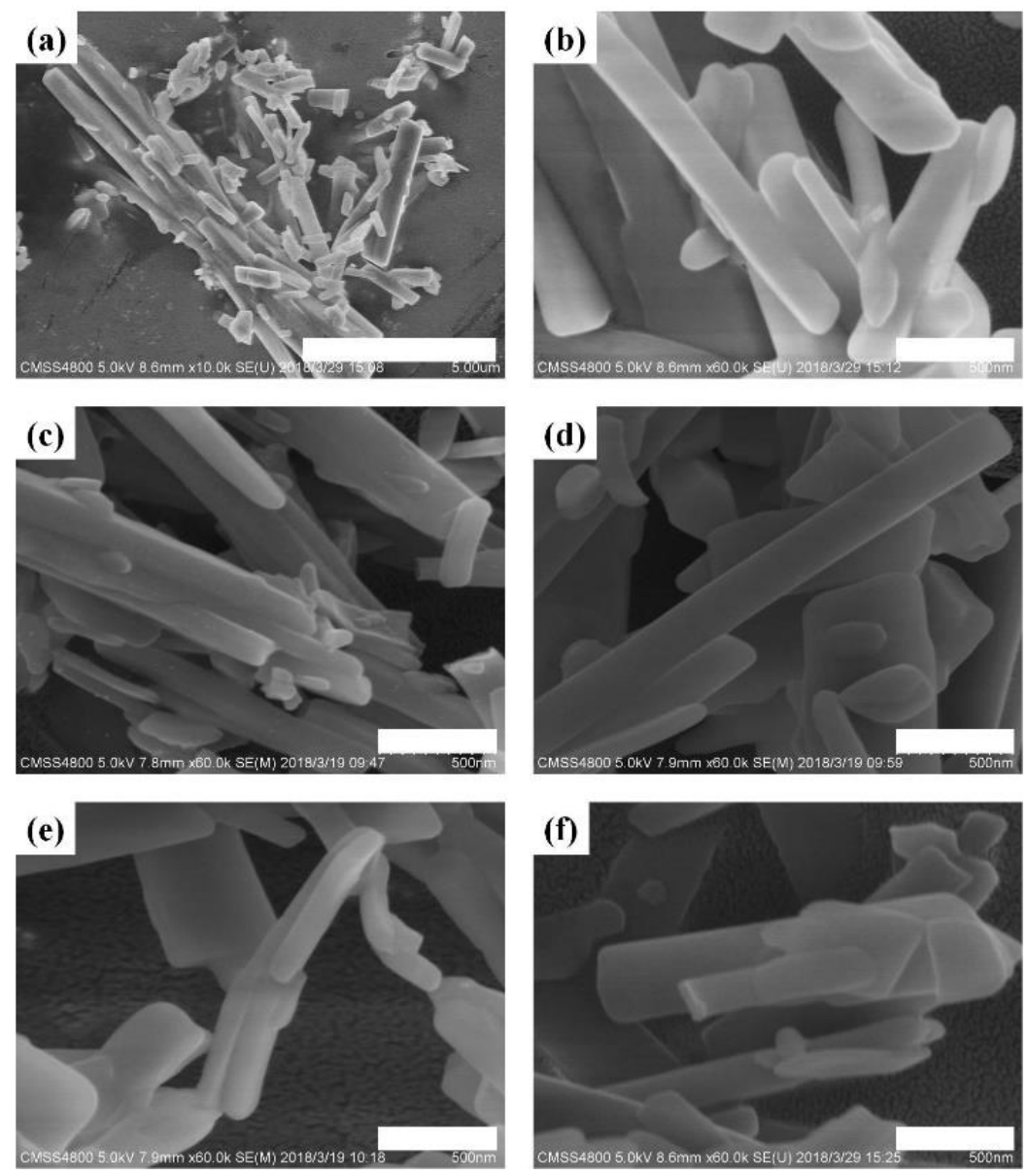

Figure 3. SEM images of the Ni-BTC samples obtained at various reaction times. (a,b) Ni-BTC-1 m, (c) Ni-BTC-5 m, (d) Ni-BTC-30m, (e) Ni-BTC-60 m, and (f) Ni-BTC-180 m. The bar in (a) represents 5 $\mu \mathrm{m}$, and the bars in (b-f) to represent $500 \mathrm{~nm}$.

\subsubsection{Effect of Mechano-Frequency of Grinding}

In addition to the grinding time, the mechano-frequency of grinding is also one key factor of ball-milling conditions. With the assist of water, the products were obtained under different frequencies from $10 \mathrm{~Hz}$ to $50 \mathrm{~Hz}$ by keeping the reaction time of only for one minute, and they denoted as Ni-BTC-10 Hz, Ni-BTC-20 Hz, Ni-BTC-30 Hz, Ni-BTC-40 Hz, and Ni-BTC-50 Hz, respectively. Their XRD patterns were shown in Figure 4a. All these patterns show the characteristic diffraction peaks of $\mathrm{Ni}_{3}(\mathrm{BTC})_{2} \cdot 12 \mathrm{H}_{2} \mathrm{O}$, even the product of Ni-BTC-10Hz. The crystallinity of the products obtained at $10 \mathrm{~Hz}$ and $20 \mathrm{~Hz}$ are also significantly lower than that obtained at higher frequencies. The yield of $\mathrm{Ni}-\mathrm{BTC}-10 \mathrm{~Hz}$ is only about $39 \%$ (Table S4). When the mechano-frequency increases to $20 \mathrm{~Hz}$, the yield increases to about $60 \%$. To further increase the milling frequency, the yield maintains at about $60-70 \%$. 

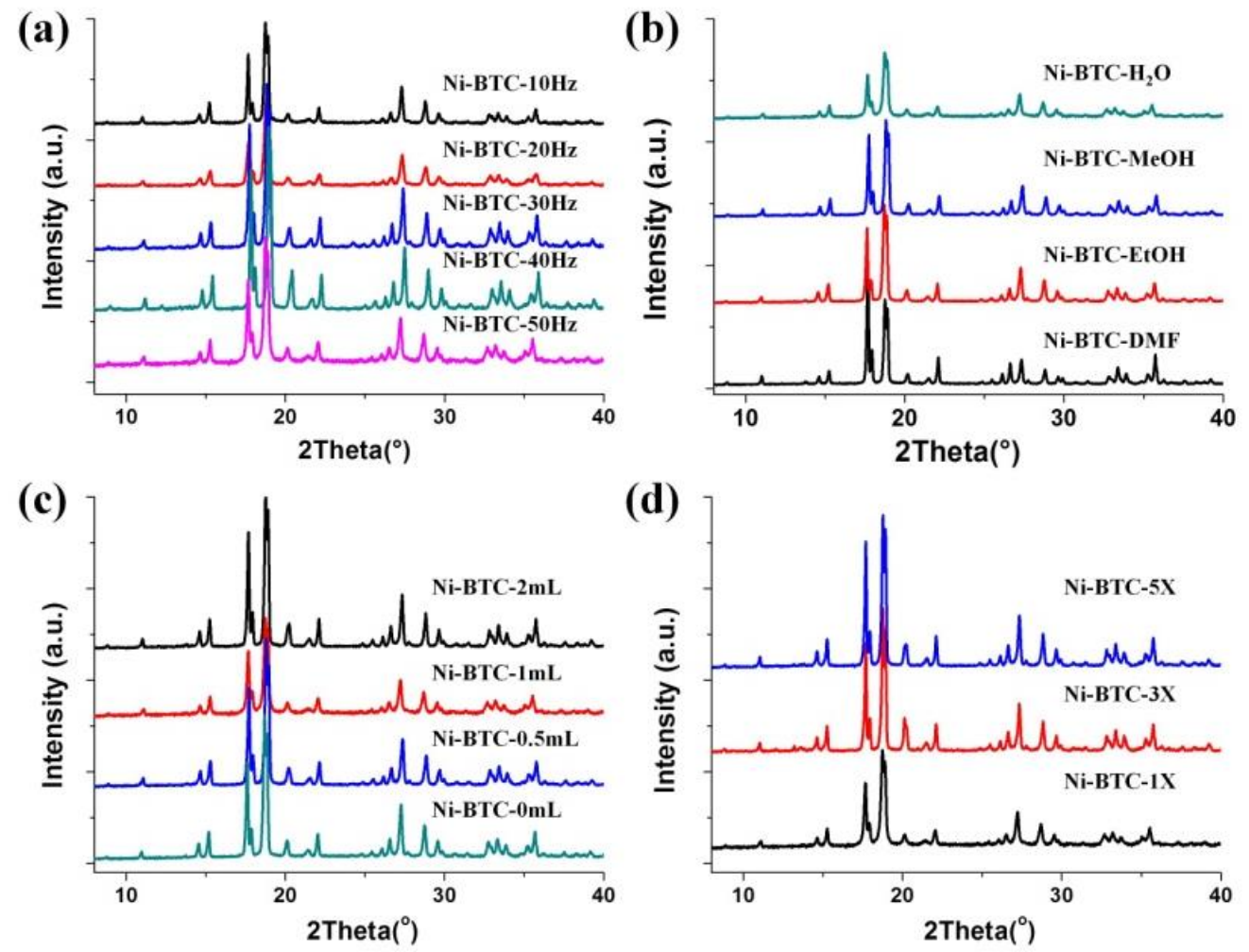

Figure 4. XRD patterns of the Ni-BTC samples obtained at various grinding frequencies (a), with addition of different solvents (b), with addition of varied amount of water (c), and at enlarged scales (d).

\subsubsection{Effect of Auxiliary Liquid}

The effect of the type of auxiliary liquid on the ball milling reaction was studied. In addition to water, the polar solvents $\mathrm{MeOH}, \mathrm{EtOH}$, and DMF, commonly used in MOF synthesis, were also explored. The XRD patterns of products were shown in Figure $4 \mathrm{~b}$. All of them have similar patterns which match well with the simulated pattern for $\mathrm{Ni}_{3}(\mathrm{BTC})_{2} \cdot 12 \mathrm{H}_{2} \mathrm{O}$, suggesting the success of preparation of product in one minute whatever the kind of auxiliary liquid. The yields of the products are between $60 \%$ to $70 \%$ (Table S5). Water is the best choice among them, considering its environment friendliness. Moreover, the effect of the quantity of water was also investigated. Unexpectedly, it is found that the product can be obtained without water, as shown in Figure $4 \mathrm{c}$, and the yield can achieve about $56 \%$ (Table S6). Under the water-assist condition, the yields increase a little to around $65 \%$. This result implies the addition of water is not necessary. Based on this result, the effects of mechano-frequency were explored again under no liquid-assist condition. In the absence of additives, even at a minimum of $10 \mathrm{~Hz}$, we can still obtain a product with good crystallization (Figure S3). However, the yield drops to only about $29 \%$ (Table S7). As the frequency increases, the yield increases slightly. Even if the frequency rises to $40 \mathrm{~Hz}$, the yield is only about $40 \%$. When the frequency is raised to $50 \mathrm{~Hz}$ (the maximum limit frequency of the instrument), the yield rises to $56 \%$. In contrast to the case with liquid assist (Figure 5), it can be seen that the yield of product with liquid assistance is significantly higher than that without liquid assistance. Under the liquid assist condition, the maximum yield can be achieved at when the frequency is over $30 \mathrm{~Hz}$, while without liquid assistance there is still a large increase in yield at $50 \mathrm{~Hz}$. 


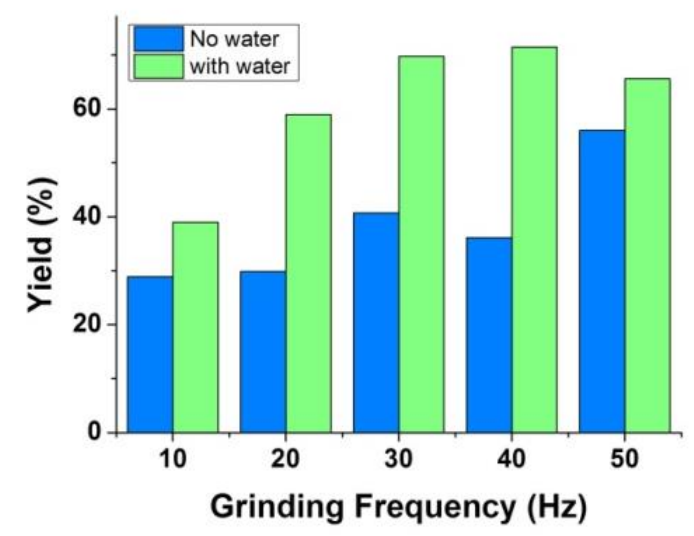

Figure 5. Comparison of yields of Ni-BTC samples obtained with and without addition of water (1 mL).

\subsubsection{Scalability}

In addition, we investigated the scalability and expandability of the method. We increased the feed rate by three times and five times, and found that the crystallinity of the product was basically the same (Figure 4d), and the yield was slightly improved (Table S8). This method can be used for bulk preparation of MOFs easily at the gram scale ( $2.77 \mathrm{~g}$ in a run). In general, the method is rapid, high-efficiency, eco-friendly, low cost, and has great scalability.

Recently, a few reports $[17,36,37]$ have also demonstrated the rapid synthesis of MOFs (Table S9). Duan et al. [36] demonstrated the synthesis of hierarchical porous ZIF-8 materials within one min at room temperature by using organic amines as a supramolecular template (organic amine-template), but the bulk organic solvent (methanol) and additives (organic amines) were still required. Jin et al. [17] synthesized $\mathrm{Ni}_{3}(\mathrm{BTC})_{2} \cdot 12 \mathrm{H}_{2} \mathrm{O}$ by a solution-phase method under room temperature in short time, but the organic linker must to be deprotonated, and the organic solvent were also used. Huang et al. [37] reported the synthesis of $\mathrm{F}_{4}$-UiO-66 in $100 \mathrm{~s}$ using water-assisted grinding, however, the metal source must be the pre-prepared zirconium clusters. Despite these developments, it should be noted that the work described here represents the first example of ultrafast synthesis of Ni-MOF by ball milling without solvent or any preliminary preparation.

This fast reaction speed is due to the sufficient energy provided by the mechanical force during ball milling. From a thermodynamic point of view, generally, the chemical potential of a substance in the solid state is higher than that of the same one in the liquid state [30,38-40]. Thus, the driving force of the formation of Ni-MOF is higher than that of solution-based synthesis method [30]. Therefore, the mechanochemical construction of Ni-MOF can be reacted within a short time. On the other hand, the additional solvents (water, $\mathrm{MeOH}, \mathrm{EtOH}$, or $\mathrm{DMF}$ ) had a good dissolution of the raw materials and, thus, they can boost molecular mobility of the reactants during reaction. Even if no addition of solvent, a small amount of crystal water contained in nickel acetate separates out under the action of ball milling, thereby acting as an auxiliary solvent. Moreover, the acetate ion acts as a base to catalyze the deprotonation of the $\mathrm{H}_{3} \mathrm{BTC}$, thereby increasing the reaction rate. In the experiment, nickel chloride and nickel nitrate were used as the metal source to carry out the reaction, and no detectable product was found, which proved the inference.

\subsection{Electrochemical Performance of Ni-MOF}

Finally, to prove that the Ni-MOF produced within one minute has considerable electrochemical performance, cyclic voltammetry (CV) and galvanostatic charge-discharge (GCD) were performed using a three electrode system in $6 \mathrm{~mol} / \mathrm{L} \mathrm{KOH}$ electrolyte and the results were presented in Figure 6. The $C V$ was carried out with a potential range from 0 to $0.5 \mathrm{~V}$ at varied scan rates $(5,10,25,50,75$, and $100 \mathrm{mV} / \mathrm{s}$ ). There are a couple of distinct redox peaks could be observed, which are correspond to the reversible redox of the reversible intercalation and deintercalation of $\mathrm{OH}^{-}$ions [13]. These surface 
faradic redox reactions lead to the typical pseudocapacitive behavior of Ni-MOFs. The GCD curves under different current densities in a potential range of $0-0.45 \mathrm{~V}$ are shown in Figure $4 \mathrm{~b}$. It can be found that there is a plateau in the potential from 0.2 to $0.25 \mathrm{~V}$ at different current densities from 1 to $10 \mathrm{~A} / \mathrm{g}$. Such a plateau is ascribed to the redox reaction, indicating the significant contribution of the pseudo-capacitance, which agrees well with the phenomena of CV curves. The specific capacitance $C_{m}$ $(\mathrm{F} / \mathrm{g})$ is calculated from the GCD measurement (Figure $4 \mathrm{~b}$ ) according to the following equation [13]:

$$
C_{\mathrm{m}}=I \Delta \mathrm{t} / \Delta E=i \Delta t /(m \Delta E)
$$

where $I$ is the discharge current density calculated using $I=i / m, i$ is the current and $m$ is the active mass of the electrode, $\Delta t$ is the during time of the discharge curve, and $\Delta E$ is the potential window of the discharge curve, respectively. The calculated $C_{\mathrm{m}}$ values of Ni-MOF- 1 are present in Figure $\mathrm{S} 4$. The specific capacitance can achieve $640 \mathrm{~F} / \mathrm{g}$ at the current density of $1 \mathrm{~A} / \mathrm{g}$, which is comparable to the performance of the hydrothermally-synthesized one [13].
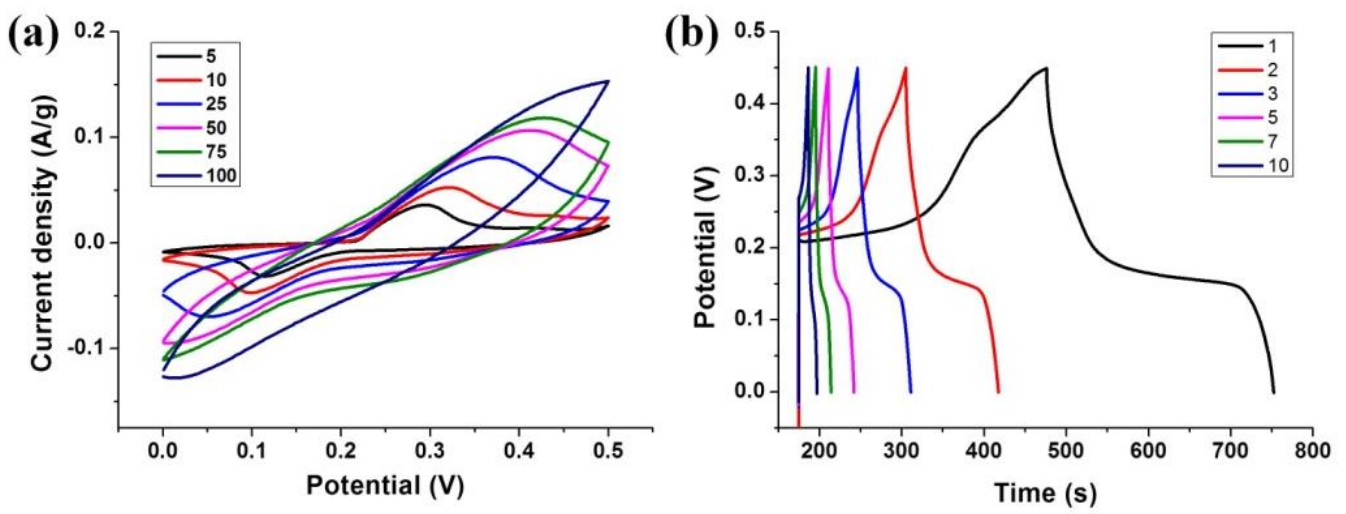

Figure 6. (a) Evolution of CVs of Ni-BTC-1 at various scan rates: 5, 10, 25, 50, 75, and $100 \mathrm{mV} / \mathrm{s}$; and (b) charge-discharge diagrams of Ni-BTC-1 at different current densities: 1, 2, 3, 5, 7, and $10 \mathrm{~A} / \mathrm{g}$.

\section{Conclusions}

We have proposed a mechanical ball milling method for ultrafast synthesis of Ni-based metal organic frameworks. The results of XRD show that there is no significant difference in the crystallinity of the products obtained at different reaction times (1-180 $\mathrm{min}$ ), and the stable product can be obtained even only at macheno-frequency of $10 \mathrm{~Hz}$ within one minute without any solvent-assist. Generally, increasing the milling time, raising the mechanical frequency, and the addition of assistant solvent will increase the yield, while the kind of assistant solvent has no evident effect on the yield. The water is the best choice among the solvents, considering the environment friendliness. The method is rapid, highly efficiency, eco-friendly, and has great scalability (at the gram scale in a run).

Supplementary Materials: The following are available online at http:/ /www.mdpi.com/2079-4991/8/12/1067/ s1; Table S1. Yields of Ni-BTC samples obtained at various reaction times; Table S2. FWHM of Ni-BTC samples obtained at various reaction times; Table S3. Surface area and pore structures of Ni-BTC samples obtained at various reaction times; Figure S1. The BJH analysis of the Ni-BTC samples obtained at various reaction times; Figure S2. SEM images of the Ni-BTC samples obtained at various reaction times. (a) Ni-BTC-5 m, (b) Ni-BTC-30 m, (c) Ni-BTC-60 m, and (d) Ni-BTC-180 m; Table S4. Yields of Ni-BTC samples obtained at various grinding frequencies with addition of $1 \mathrm{~mL}$ water; Table S5. Yields of Ni-BTC samples obtained with addition of different solvents; Table S6. Yields of Ni-BTC samples obtained with addition of varied amount of water; Figure S3. XRD patterns of the Ni-BTC samples obtained at various grinding frequencies without the water addition; Table S7. Yields of Ni-BTC samples obtained at various grinding frequencies without the addition of water; Table S8. Yields of Ni-BTC samples obtained at enlarged scales; Table S9. Comparison of the synthesis of MOF in previously published reports; Figure S4. Diagram of the specific capacitance of materials at different current densities. 
Author Contributions: Conceptualization: C.-A.T.; data curation: R.Z. and L.W.; formal analysis: R.Z. and R.C.; funding acquisition: C.-A.T.; investigation: R.Z. and R.C.; methodology: C.-A.T. and J.W.; project administration: J.W.; supervision: C.-A.T. and X.Z.; visualization: L.W.; writing-original draft: C.-A.T.; writing一review and editing: X.Z. and J.W.

Funding: The National Natural Science Foundation of China (21573285), the Natural Science Foundation of Hunan Province (2018JJ3597), and a research project of National University of Defense Technology (ZK16-03-51).

Conflicts of Interest: The authors declare no conflict of interest.

\section{References}

1. Hendon, C.H.; Rieth, A.J.; Korzyński, M.D.; Dincă, M. Grand challenges and future opportunities for metal-organic frameworks. ACS Central Sci. 2017, 3, 554-563. [CrossRef] [PubMed]

2. Furukawa, H.; Cordova, K.E.; O'keeffe, M.; Yaghi, O.M. The chemistry and applications of metal-organic frameworks. Science 2013, 341, 1230444. [CrossRef] [PubMed]

3. Alezi, D.; Belmabkhout, Y.; Suyetin, M.; Bhatt, P.M.; Weselinski, Ł.J.; Solovyeva, V.; Adil, K.; Spanopoulos, I.; Trikalitis, P.N.; Emwas, A.M. Mof crystal chemistry paving the way to gas storage needs: Aluminum-based soc-mof for $\mathrm{CH}_{4}, \mathrm{O}_{2}$, and $\mathrm{CO}_{2}$ storage. J. Am. Chem. Soc. 2015, 137, 13308-13318. [CrossRef] [PubMed]

4. Kang, Z.; Fan, L.; Sun, D. Recent advances and challenges of metal-organic framework membranes for gas separation. J. Mater. Chem. 2017, 5, 10073-10091. [CrossRef]

5. Huang, Y.-B.; Liang, J.; Wang, X.-S.; Cao, R. Multifunctional metal-organic framework catalysts: Synergistic catalysis and tandem reactions. Chem. Soc. Rev. 2017, 46, 126-157. [CrossRef] [PubMed]

6. Hu, Z.; Tao, C.-A.; Liu, H.; Zou, X.; Zhu, H.; Wang, J. Fabrication of an $\mathrm{NH}_{2}$-MIL-88B photonic film for naked-eye sensing of organic vapors. J. Mater. Chem. A 2014, 2, 14222-14227. [CrossRef]

7. Lustig, W.P.; Mukherjee, S.; Rudd, N.D.; Desai, A.V.; Li, J.; Ghosh, S.K. Metal-organic frameworks: Functional luminescent and photonic materials for sensing applications. Chem. Soc. Rev. 2017, 46, 3242-3285. [CrossRef] [PubMed]

8. Liu, L.; Zhou, Y.; Liu, S.; Xu, M. The applications of metal-organic frameworks in electrochemical sensors. ChemElectroChem 2018, 5, 6-19. [CrossRef]

9. Wang, L.; Han, Y.; Feng, X.; Zhou, J.; Qi, P.; Wang, B. Metal-organic frameworks for energy storage: Batteries and supercapacitors. Coord. Chem. Rev. 2016, 307, 361-381. [CrossRef]

10. Zheng, S.; Li, X.; Yan, B.; Hu, Q.; Xu, Y.; Xiao, X.; Xue, H.; Pang, H. Transition-metal (Fe, Co, Ni) based metal-organic frameworks for electrochemical energy storage. Adv. Energy Mater. 2017, 7, 1602733. [CrossRef]

11. Zhao, Y.; Song, Z.; Li, X.; Sun, Q.; Cheng, N.; Lawes, S.; Sun, X. Metal organic frameworks for energy storage and conversion. Energy Storage Mater. 2016, 2, 35-62. [CrossRef]

12. Yang, J.; Xiong, P.; Zheng, C.; Qiu, H.; Wei, M. Metal-organic frameworks: A new promising class of materials for a high performance supercapacitor electrode. J. Mater. Chem. A 2014, 2, 16640-16644. [CrossRef]

13. Kang, L.; Sun, S.-X.; Kong, L.-B.; Lang, J.-W.; Luo, Y.-C. Investigating metal-organic framework as a new pseudo-capacitive material for supercapacitors. Chin. Chem. Lett. 2014, 25, 957-961. [CrossRef]

14. Wang, D.; Ni, W.; Pang, H.; Lu, Q.; Huang, Z.; Zhao, J. Preparation of mesoporous NiO with a bimodal pore size distribution and application in electrochemical capacitors. Electrochim. Acta 2010, 55, 6830-6835. [CrossRef]

15. Chen, L.; Bai, J.; Wang, C.; Pan, Y.; Scheer, M.; You, X. One-step solid-state thermolysis of a metal-organic framework: A simple and facile route to large-scale of multiwalled carbon nanotubes. Chem. Commun. 2008, 1581-1583. [CrossRef] [PubMed]

16. Du, P.; Dong, Y.; Liu, C.; Wei, W.; Liu, D.; Liu, P. Fabrication of hierarchical porous nickel based metal-organic framework (Ni-MOF) constructed with nanosheets as novel pseudo-capacitive material for asymmetric supercapacitor. J. Colloid Interface Sci. 2018, 518, 57-68. [CrossRef] [PubMed]

17. Jin, L.-N.; Liu, Q.; Sun, W.-Y. Room temperature solution-phase synthesis of flower-like nanostructures of $\left[\mathrm{Ni}_{3}(\mathrm{BTC})_{2} \cdot 12 \mathrm{H}_{2} \mathrm{O}\right.$ ] and their conversion to porous NiO. Chin. Chem. Lett. 2013, 24, 663-667. [CrossRef]

18. Do, J.-L.; Friščić, T. Mechanochemistry: A force of synthesis. ACS Central Sci. 2017, 3, 13-19. [CrossRef] [PubMed] 
19. James, S.L.; Adams, C.J.; Bolm, C.; Braga, D.; Collier, P.; Friščić, T.; Grepioni, F.; Harris, K.D.M.; Hyett, G.; Jones, W.; et al. Mechanochemistry: Opportunities for new and cleaner synthesis. Chem. Soc. Rev. 2012, 41, 413-447. [CrossRef] [PubMed]

20. Friščić, T. Supramolecular concepts and new techniques in mechanochemistry: Cocrystals, cages, rotaxanes, open metal-organic frameworks. Chem. Soc. Rev. 2012, 41, 3493-3510. [CrossRef] [PubMed]

21. Pichon, A.; James, S.L. An array-based study of reactivity under solvent-free mechanochemical conditions-Insights and trends. CrystEngComm 2008, 10, 1839-1847. [CrossRef]

22. Beldon, P.J.; Fábián, L.; Stein, R.S.; Thirumurugan, A.; Cheetham, A.K.; Friščić, T. Rapid room-temperature synthesis of zeolitic imidazolate frameworks by using mechanochemistry. Angew. Chem. 2010, 122, 9834-9837. [CrossRef]

23. Katsenis, A.D.; Puškarić, A.; Štrukil, V.; Mottillo, C.; Julien, P.A.; Užarević, K.; Pham, M.-H.; Do, T.-O.; Kimber, S.A.J.; Lazić, P.; et al. In situ X-ray diffraction monitoring of a mechanochemical reaction reveals a unique topology metal-organic framework. Nat. Commun. 2015, 6, 6662. [CrossRef] [PubMed]

24. Yuan, W.; O'Connor, J.; James, S.L. Mechanochemical synthesis of homo- and hetero-rare-earth(iii) metal-organic frameworks by ball milling. CrystEngComm 2010, 12, 3515-3517. [CrossRef]

25. Prochowicz, D.; Sokołowski, K.; Justyniak, I.; Kornowicz, A.; Fairen-Jimenez, D.; Friščić, T.; Lewiński, J. A mechanochemical strategy for IRMOF assembly based on pre-designed oxo-zinc precursors. Chem. Commun. 2015, 51, 4032-4035. [CrossRef] [PubMed]

26. Pilloni, M.; Padella, F.; Ennas, G.; Lai, S.; Bellusci, M.; Rombi, E.; Sini, F.; Pentimalli, M.; Delitala, C.; Scano, A.; et al. Liquid-assisted mechanochemical synthesis of an iron carboxylate metal organic framework and its evaluation in diesel fuel desulfurization. Microp. Mesoporous Mater. 2015, 213, 14-21. [CrossRef]

27. Julien, P.A.; Užarević, K.; Katsenis, A.D.; Kimber, S.A.J.; Wang, T.; Farha, O.K.; Zhang, Y.; Casaban, J.; Germann, L.S.; Etter, M.; et al. In situ monitoring and mechanism of the mechanochemical formation of a microporous MOF-74 framework. J. Am. Chem. Soc. 2016, 138, 2929-2932. [CrossRef] [PubMed]

28. Stolar, T.; Batzdorf, L.; Lukin, S.; Žilić, D.; Motillo, C.; Friščić, T.; Emmerling, F.; Halasz, I.; Užarević, K. In situ monitoring of the mechanosynthesis of the archetypal metal-organic framework HKUST-1: Effect of liquid additives on the milling reactivity. Inorg. Chem. 2017, 56, 6599-6608. [CrossRef] [PubMed]

29. Liu, W.; Zhu, K.; Teat, S.J.; Deibert, B.J.; Yuan, W.; Li, J. A mechanochemical route toward the rational, systematic, and cost-effective green synthesis of strongly luminescent copper iodide based hybrid phosphors. J. Mater. Chem. C 2017, 5, 5962-5969. [CrossRef]

30. Chen, Y.; Wu, H.; Liu, Z.; Sun, X.; Xia, Q.; Li, Z. Liquid-assisted mechanochemical synthesis of copper based MOF-505 for the separation of $\mathrm{CO}_{2}$ over $\mathrm{CH}_{4}$ or $\mathrm{N}_{2}$. Ind. Eng. Chem. Res. 2018, 57, 703-709. [CrossRef]

31. Užarević, K.; Wang, T.C.; Moon, S.-Y.; Fidelli, A.M.; Hupp, J.T.; Farha, O.K.; Friščić, T. Mechanochemical and solvent-free assembly of zirconium-based metal-organic frameworks. Chem. Commun. 2016, 52, 2133-2136. [CrossRef] [PubMed]

32. Fidelli, A.M.; Karadeniz, B.; Howarth, A.J.; Huskić, I.; Germann, L.S.; Halasz, I.; Etter, M.; Moon, S.-Y.; Dinnebier, R.E.; Stilinović, V.; et al. Green and rapid mechanosynthesis of high-porosity NU- and UiO-type metal-organic frameworks. Chem. Commun. 2018, 54, 6999-7002. [CrossRef] [PubMed]

33. Ali-Moussa, H.; Navarro Amador, R.; Martinez, J.; Lamaty, F.; Carboni, M.; Bantreil, X. Synthesis and post-synthetic modification of UiO-67 type metal-organic frameworks by mechanochemistry. Mater. Lett. 2017, 197, 171-174. [CrossRef]

34. Yaghi, O.M.; Li, H.; Groy, T.L. Construction of porous solids from hydrogen-bonded metal complexes of 1,3,5-benzenetricarboxylic acid. J. Am. Chem. Soc. 1996, 118, 9096-9101. [CrossRef]

35. CCDC No. 1274034. Available online: http://www.ccdc.cam.ac.uk/access (accessed on 17 December 2017).

36. Duan, C.; Li, F.; Xiao, J.; Liu, Z.; Li, C.; Xi, H. Rapid room-temperature synthesis of hierarchical porous zeolitic imidazolate frameworks with high space-time yield. Sci. China Mater. 2017, 60, 1205-1214. [CrossRef]

37. Huang, Y.-H.; Lo, W.-S.; Kuo, Y.-W.; Chen, W.-J.; Lin, C.-H.; Shieh, F.-K. Green and rapid synthesis of zirconium metal-organic frameworks via mechanochemistry: UiO-66 analog nanocrystals obtained in one hundred seconds. Chem. Commun. 2017, 53, 5818-5821. [CrossRef] [PubMed]

38. Chen, Y.; Xiao, J.; Lv, D.; Huang, T.; Xu, F.; Sun, X.; Xi, H.; Xia, Q.; Li, Z. Highly efficient mechanochemical synthesis of an indium based metal-organic framework with excellent water stability. Chem. Eng. Sci. 2017, 158, 539-544. [CrossRef] 
39. Yang, H.; Orefuwa, S.; Goudy, A. Study of mechanochemical synthesis in the formation of the metal-organic framework $\mathrm{Cu}_{3}(\mathrm{BTC})_{2}$ for hydrogen storage. Microp. Mesoporous Mater. 2011, 143, 37-45. [CrossRef]

40. Bowmaker, G.A. Solvent-assisted mechanochemistry. Chem. Commun. 2013, 49, 334-348. [CrossRef] [PubMed]

(c)

(c) 2018 by the authors. Licensee MDPI, Basel, Switzerland. This article is an open access article distributed under the terms and conditions of the Creative Commons Attribution (CC BY) license (http:/ / creativecommons.org/licenses/by/4.0/). 